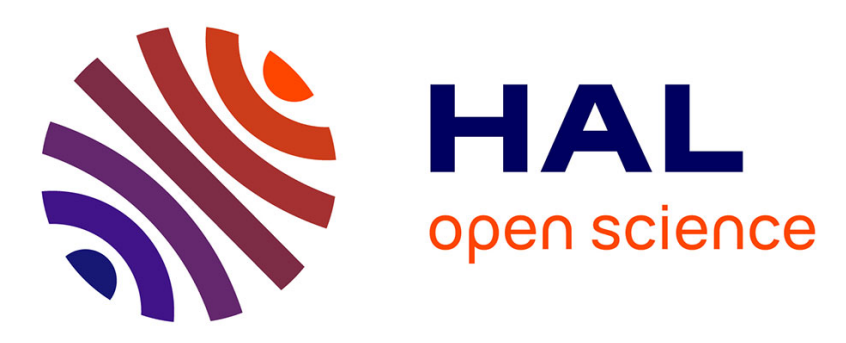

\title{
Hybrid systems with Ag nanocrystals and Si nanostructures synthesized by ultra-low-energy ion beam synthesis
}

Marzia Carrada, Assia Haj Salem, Béatrice Pécassou, Robert Carles, Gérard Benassayag

\section{To cite this version:}

Marzia Carrada, Assia Haj Salem, Béatrice Pécassou, Robert Carles, Gérard Benassayag. Hybrid systems with Ag nanocrystals and Si nanostructures synthesized by ultra-low-energy ion beam synthesis. Journal of Applied Physics, 2017, 122 (10), pp.103101. 10.1063/1.5000360 . hal-01755324

\section{HAL Id: hal-01755324 https://hal.science/hal-01755324}

Submitted on 5 Apr 2018

HAL is a multi-disciplinary open access archive for the deposit and dissemination of scientific research documents, whether they are published or not. The documents may come from teaching and research institutions in France or abroad, or from public or private research centers.
L'archive ouverte pluridisciplinaire HAL, est destinée au dépôt et à la diffusion de documents scientifiques de niveau recherche, publiés ou non, émanant des établissements d'enseignement et de recherche français ou étrangers, des laboratoires publics ou privés. 


\title{
Hybrid systems with Ag nanocrystals and Si nanostructures synthesized by ultra-low- energy ion beam synthesis
}

\author{
M. Carrada, A. Haj Salem, B. Pecassou, R. Carles, and G. Ben Assayag
}

Citation: Journal of Applied Physics 122, 103101 (2017); doi: 10.1063/1.5000360

View online: https://doi.org/10.1063/1.5000360

View Table of Contents: http://aip.scitation.org/toc/jap/122/10

Published by the American Institute of Physics

\section{Articles you may be interested in}

Role of the Fermi level in the formation of electronic band-tails and mid-gap states of hydrogenated amorphous silicon in thin-film solar cells

Journal of Applied Physics 122, 093101 (2017); 10.1063/1.4989425

Imaging optical fields below metal films and metal-dielectric waveguides by a scanning microscope Journal of Applied Physics 122, 113101 (2017); 10.1063/1.5002071

Structural and optical characterization of AIGaN multiple quantum wells grown on semipolar (20-21) bulk AIN substrate

Applied Physics Letters 111, 111101 (2017); 10.1063/1.4985156

Detection of guided-wave plasmon polariton modes in a high-index dielectric MIM structure Journal of Applied Physics 122, 123101 (2017); 10.1063/1.5001902

High performance terahertz metasurface quantum-cascade VECSEL with an intra-cryostat cavity Applied Physics Letters 111, 101101 (2017); 10.1063/1.4993600

Massive parallel 3D PIC simulation of negative ion extraction

Journal of Applied Physics 122, 103302 (2017); 10.1063/1.5001397

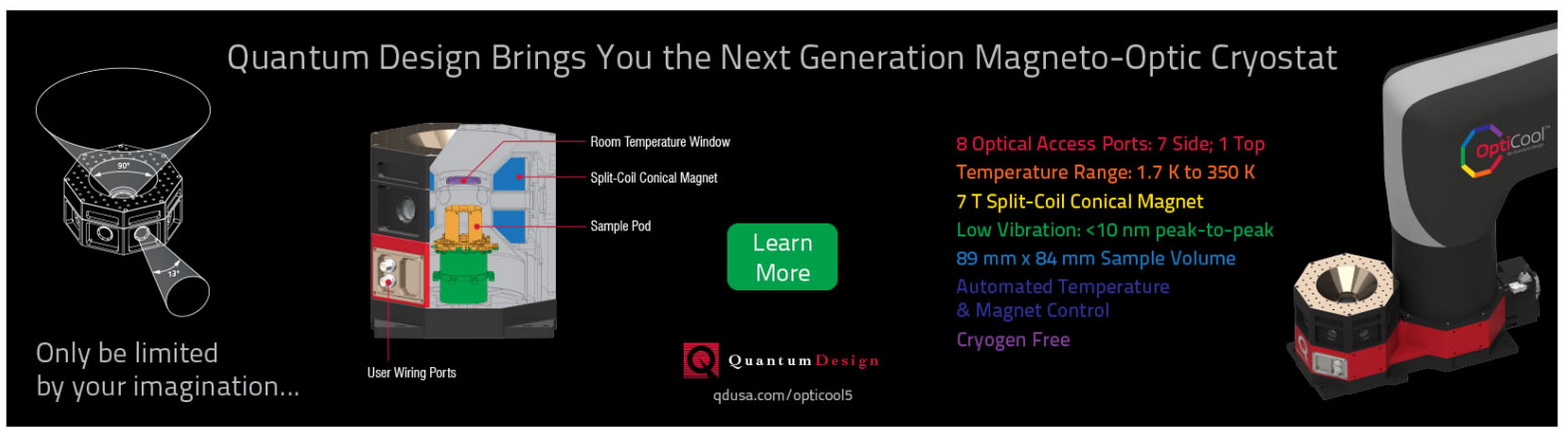




\title{
Hybrid systems with Ag nanocrystals and Si nanostructures synthesized by ultra-low-energy ion beam synthesis
}

\author{
M. Carrada, ${ }^{\text {a) }}$ A. Haj Salem, B. Pecassou, R. Carles, and G. Ben Assayag \\ CEMES-CNRS, Université de Toulouse, 29 rue J. Marvig, 31055 Toulouse Cedex 04, France
}

(Received 6 March 2017; accepted 15 August 2017; published online 8 September 2017)

\begin{abstract}
Hybrid systems based on silicon and silver nanocrystals (Si-NCs and Ag-NCs) are of considerable interest in photon conversion solar cells. Due to their plasmonic properties, Ag-NCs strongly increase the photoluminescence emission intensity of Si-NCs located in their vicinity, allowing, in principle, to solve the problem of their low emission yield. In this work, we have elaborated 2D networks of Ag-NCs and amorphous Si nanoparticles in a controlled way by using Ultra-LowEnergy Ion-Beam-Synthesis. In the proposed synthesis scheme, a 2D layer of Si-NCs is first obtained by implanting $\mathrm{Si}^{+}$ions at ultra low energy (from 1 to $3 \mathrm{keV}$ ) in a $\mathrm{SiO}_{2}$ layer with subsequent high temperature thermal annealing. Then, $\mathrm{Ag}^{+}$ions are implanted in the same matrix at energies between 3 and $10 \mathrm{keV}$ and crystalline Ag-NCs are formed during the implantation step. Several configurations with either 2D arrays or a large band of Ag-NCs have been obtained following the $\mathrm{Ag}^{+}$implantation energy. Enhancement of the PL emission from Si nanostructures, which is related to the presence of Ag-NCs, has been observed under specific arrangement of the two embedded subsystems. In this type of synthesis, a combination of physical phenomena including ion mixing, implantation damage, point defect, and thermal diffusion has been taken into account in order to explain and thus control the structural and the optical characteristics of the system. Published by AIP Publishing. [http://dx.doi.org/10.1063/1.5000360]
\end{abstract}

\section{INTRODUCTION}

Hybrid systems composed of metallic (silver) and semiconductor (silicon) nanoparticles embedded in dielectric matrix have been studied in recent years for photovoltaic (PV) applications. Indeed, one of the approaches of 3rd generation PV consists in adapting the incident light on a standard solar cell with a photon converter material in order to increase the efficiency of photovoltaic conversion. ${ }^{1,2}$ In these hybrid systems, silicon nanocrystals ( $\mathrm{Si}-\mathrm{NCs}$ ) are used as photon converters (or down-shifters) due to their luminescent properties in the red region of the light spectrum, while $\mathrm{Ag}$ nanocrystals (Ag-NCs) are coupled to them in order to enhance photoluminescence (PL) emission, thanks to their plasmonics properties. $^{3-5}$

The optimization of the coupling in Si-NCs/Ag-NCs systems strongly depends on the structural characteristics of the two types of nanoparticles, and then it is essential to control their fabrication at the nanoscale.

Several approaches have been developed to fabricate such hybrid systems. In most of the cases reported in the literature, the fabrication of the two kinds of nanoparticles is obtained by using two different synthesis techniques. For example, some groups obtained $\mathrm{Si}-\mathrm{NCs}$ by plasma enhanced chemical vapour deposition (PECVD), thanks to Si rich $\mathrm{SiN}_{\mathrm{x}}$ layers deposition followed by high temperature $\left(>900^{\circ} \mathrm{C}\right)$ annealing, while $\mathrm{Ag}-\mathrm{NCs}$ were subsequently obtained by other methods, such as electroless deposition ${ }^{6}$ or e-beam evaporation. ${ }^{7}$ Other groups synthesized $\mathrm{Si}-\mathrm{NCs}$ by ion implantation while Ag-NCs were obtained later by electron

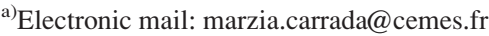

lithography ${ }^{8-10}$ or by magnetron sputtering. ${ }^{11}$ We could only find one group who studied a system containing Si-NCs and Ag-NCs both synthesized by ion implantation, ${ }^{12,13}$ but they used very high-energy ions in the $\mathrm{MeV}$ range. The structural characteristics of the obtained sample are not described in these studies, but the observed PL emission, which is enhanced after Ag ion implantation, was attributed to $\mathrm{Si}$ NCs. However, as the energy used by these last authors is very high, the particle sizes and their spatial broadening are expected to follow the Gaussian and very wide distribution of the implanted ions.

In this paper, we present a method, which, in principle, allows obtaining $\mathrm{Si}-\mathrm{NCs}$ and $\mathrm{Ag}-\mathrm{NCs}$ in a controlled way by exclusively using ion implantation. We propose to use Ultra Low Energy-Ion Beam Synthesis (ULE-IBS) to obtain an ideal system made of only one plane of each type of nanoparticles. Indeed, ULE-IBS is a well-known method for the fabrication of controlled nanostructures: in the past, we have successfully synthesized 2D arrays of Si-NCs ${ }^{14-17}$ and more recently of $\mathrm{Ag}-\mathrm{NCs}^{18,19}$ with well controlled distances and sizes distributions.

In this work, we have investigated the ability of dual ULE-IBS to synthesize 2D arrays of Si-NCs and Ag-NCs in the same dielectric matrix. The possibility to tune the mutual distances of the 2D arrays of the two species of nanoparticles should ease a systematic study of the coupling of Si-NCs and Ag-NCs.

Our synthesis process for $\mathrm{Si}$ and Ag nanocrystals 2D arrays consists in two steps ULE-IBS. The first one consists in forming the $\mathrm{Si}-\mathrm{NCs}$ that requires high temperature thermal annealing $\left(1050^{\circ} \mathrm{C}\right)$. Then, $\mathrm{Ag}-\mathrm{NCs}$ are obtained by subsequent silver ion implanting into the matrix containing 
Si-NCs. After this step, Si-NCs are amorphised and the population characteristics changes. Many physical phenomena are involved in this type of synthesis including ion mixing, damage, and diffusion. The aim of this work is to investigate these phenomena and to clarify which are the conditions to synthesize controlled bi-layers of Si-NCs and Ag-NCs.

\section{EXPERIMENTAL DETAILS}

The investigated samples have been fabricated by using by the following method: $240 \mathrm{~nm}$ thick $\mathrm{SiO}_{2}$ layers thermally grown on (100) Si wafers have been implanted in a modified Varian 200-A2F ion implanter with ${ }^{28} \mathrm{Si}^{+}$with energies from 1 to $3 \mathrm{keV}$ and fluences of $5 \times 10^{15}$ up to $2 \times 10^{16} \mathrm{~cm}^{-2}$. After the implantation step, the samples were RTA (Rapid Thermal Annealing) annealed for $1 \mathrm{~min}$ at $1050^{\circ} \mathrm{C}$ under inert $\left(\mathrm{N}_{2}\right)$ ambient. Then, these samples were implanted with ${ }^{107} \mathrm{Ag}^{+}$ions with energies from 3 up to $10 \mathrm{keV}$ and fluences between $4.7 \times 10^{15}$ and $7.15 \times 10^{15} \mathrm{~cm}^{-2}$.

Specimens from all samples were prepared for crosssectional TEM observations using a Zeiss/OrsayPhysics XB1540 dual beam Focused Ion Beam (FIB) equipment.

The double layers were observed by High Resolution Transmission Electron Microscopy (HREM) and by Energy Filtered Transmission Electron Microscopy (EFTEM). EFTEM is the most appropriate technique to image $\mathrm{Si}-\mathrm{NCs}$ in a silicon oxide matrix, independently of their amorphous or crystallized state and orientation. This method associates electron energy-loss spectroscopy (EELS) to TEM in order to achieve chemical mapping; energy-filtered images are recorded using a selection slit positioned at an energy of $17 \mathrm{eV}$ (corresponding to the Si plasmon energy) and a width equivalent to an energy window of $4 \mathrm{eV}$. The contrast between Silicon and Silica can be obtained, thanks to the difference in their plasmon energies (17 and $23 \mathrm{eV}$ respectively). However, due to the partial overlap of their plasmon profiles, Si-NCs appear bright as the silica matrix is grey in this kind of images. Even if Ag-NCs can be observed in silica with conventional Bright Field TEM, we have chosen to show only EFTEM images of the samples because, in this case, both $\mathrm{Si}$ and $\mathrm{Ag}-\mathrm{NCs}$ can be simultaneously seen. Indeed, $\mathrm{Ag}-\mathrm{NCs}$ contrast appears dark because no Ag signal is present in the EELS spectra at the energy used to obtain the filtered images.

EFTEM and HREM observations were taken on crosssectional specimens using a FEI Tecnai ${ }^{\mathrm{TM}}$ F20 field emission TEM, operating at $200 \mathrm{kV}$, equipped with a spherical aberration corrector and a TRIDIEM Gatan Imaging Filter (GIF).

In order to predict the target stoichiometry after $\mathrm{Si}$ and $\mathrm{Ag}$ implantation, two Monte Carlo simulation programs based on the binary collision approximation have been used. The SRIM software (Stopping and Range of Ions in Matter) based on the TRIM code, was used to calculate, mainly, the damage distribution. Compared to SRIM, the code TRYDIN includes dynamic target changes for high fluences during ion implantation and was used to predict the ion sputtering, and ion beam mixing.

Finally, the PL spectra were recorded at room temperature using a Jobin-Yvon XploRA set-up using the $532 \mathrm{~nm}$ laser line excitation.

\section{RESULTS AND DISCUSSION}

The aim of this work is to investigate the ability to fabricate double 2D layers of Si- and Ag-NCs by ULE-IBS and to finely control their characteristics. The study of double layers of nanocrystals finds its roots in our past experience on the synthesis by ULE-IBS of either Si-NCs or Ag-NCs in a silica matrix. Thanks to our previous works we are able to control the characteristics of the nanocrystals which are disposed on a plane (position with respect to the surface, sizes, and densities) and this, for both the species separately. Concerning the implantation energy $E$, for example, we know that in order to obtain 2D arrays of nanocrystals (distribution on a plane parallel to the sample surface), it is necessary to limit this energy $E \leq 3 \mathrm{keV}$ in the case of $\mathrm{Si}^{-\mathrm{NCs}^{14}}$ and to $E \leq 10 \mathrm{keV}$ for Ag-NCs. ${ }^{19}$ For higher energies, the ion concentration profile is larger, and the nanocrystals are distributed in a wider band. Additionally, previous works on $\mathrm{Ag}-\mathrm{NCs}$ have also shown that the nanoparticles obtained at the lowest energies available in our implanter, about $1 \mathrm{keV}$, are formed very close to the surface, but their size and densities are very low due to sputtering effects. ${ }^{19}$ Moreover, small particles are known to absorb the incident light, thus making the coupling with $\mathrm{Si}-\mathrm{NCs}$ inefficient. For these reasons, very low energy for Ag-NCs synthesis $(E \leq 1 \mathrm{keV})$ is not suitable for optimised double layers synthesis and will not be investigated here.

Figure 1 shows the $\mathrm{Si}$ and $\mathrm{Ag}$ ion concentration distributions obtained by TRIM simulations for Ultra Low Energy Ion Implantation in a $\mathrm{SiO}_{2}$ matrix with various energies. These calculated profiles show that for the same kinetic energy, $\mathrm{Si}$ atoms projected range $(R p)$ is lower than $\mathrm{Ag}$ one, suggesting that it will be easier to obtain double layers with $\mathrm{Si}-\mathrm{NCs}$ closer to the sample surface, while Ag-NCs should be synthesized deeper in the layer. However, the order in which the nanocrystals are synthesized is imposed by the thermal budget necessary for their nucleation and growth. In our system, due a very low diffusion coefficient of Si in silica, Si-NCs have to be synthesized first as the annealing temperature is very high $\left(\mathrm{T}>900^{\circ} \mathrm{C}\right)$ while $\mathrm{Ag}$-NCs are formed and well crystallized during the implantation step. For these

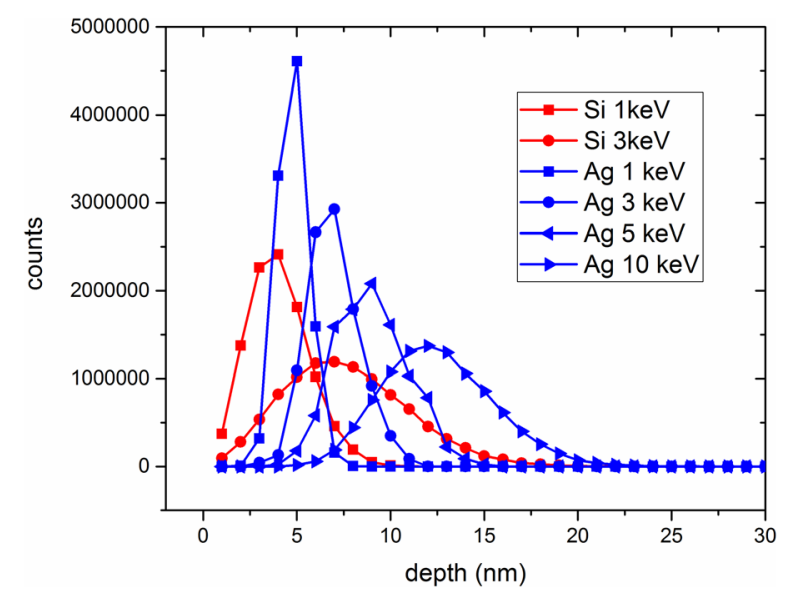

FIG. 1. Si (in red) and Ag (in blue) ion concentration distributions obtained by TRIM simulations for Ultra-Low-Energy Ion Implantation in a $\mathrm{SiO}_{2}$ matrix, with energies between 1 and $3 \mathrm{keV}$ for $\mathrm{Si}$ and 1 and $10 \mathrm{keV}$ for $\mathrm{Ag}$. 
reasons, the synthesis of double layers is much more complicated than a single layer one. In fact, as $\mathrm{Si}-\mathrm{NCs}$, which are formed close to the sample surface, have to be synthetized first, it is necessary to implant the silver through the already formed Si-NCs, which will induce some ion mixing in the Si-NCs layers.

Our primary approach for double layers synthesis consists of three stages: first, $\mathrm{Si}$ ions are implanted with energy $\leq 3 \mathrm{keV}$, then the samples are RTA annealed at $1050^{\circ} \mathrm{C}$ under $\mathrm{N}_{2}$ ambient for $60 \mathrm{~s}$ to obtain Si-NCs. The third stage consists in implanting $\mathrm{Ag}^{+}$ions to synthesize Ag-NCs.

In this work, we have investigated the synthesis of $2 \mathrm{D}$ arrays of $\mathrm{Si}$ and $\mathrm{Ag}$ nanocrystals by ULE-IBS, by taking into account the limitations due to the different thermal budgets for nucleation and growth for the two implanted species and the complex physical phenomena that takes place. Among these phenomena, special attention should be paid to understand and control the mixing process occurring when Ag$\mathrm{NCs}$ are formed in the $\mathrm{SiO}_{2}$ matrix containing Si-NCs. This ion-mixing might also play a role in the Ag-NCs synthesis through the host matrix modification.

In order to evaluate the impact of Ag-NCs synthesis on the Si-NCs previously formed in the matrix, we have performed two steps TRIDYN simulations in which $\mathrm{Si}$, then $\mathrm{Ag}$, are implanted into $\mathrm{SiO}_{2}$ containing a $\mathrm{Si}$ excess distributed following the previous calculated profile. According to the Silicon very low diffusivity in silica, using the implanted $\mathrm{Si}$ profile instead of Si nanocrystals is a suitable approximation. In addition, as our samples are annealed and contain Si-NCs and not a distribution of $\mathrm{Si}$ atoms profile when $\mathrm{Ag}$ is implanted, we have also simulated an $\mathrm{Ag}$ implantation through a Si thin layer within the silica matrix. However, we have to mention that preliminary results have shown that no difference is observed for Ag-NCs populations (position and size) for the same Si previously implanted conditions in the case of annealed and not annealed samples, i.e., for samples containing Si-NCs or only $\mathrm{Si}$ excess in the matrix. The aim of these TRIDYN simulations is to predict the final distribution of $\mathrm{Si}$ ions after $\mathrm{Ag}$ implantation, and at the same time to estimate the effect of the presence of Si-NCs on the Ag ions distribution.

\section{A. The case of Ag-NCs synthesized at $E=10 \mathrm{keV}$}

As we would like to obtain separated layers of Si-NCs and $\mathrm{Ag}-\mathrm{NCs}$, we started to investigate samples in which $\mathrm{Ag}$ has been implanted with $E=10 \mathrm{keV}$ corresponding to the deepest position for a 2D array of Ag-NCs.

The TRIDYN results have been reported in Fig. 2 in the case of (a) $\mathrm{SiO}_{2}$ implanted with $\mathrm{Si}$ at $1 \mathrm{keV}$ and with a fluence of $5 \times 10^{15} \mathrm{~cm}^{-2}$, (b) $\mathrm{SiO}_{2}$ implanted with $\mathrm{Si}$ at $3 \mathrm{keV}$ and with a fluence of $2 \times 10^{16} \mathrm{~cm}^{-2}$ and (c) $\mathrm{SiO}_{2}$ containing a $4 \mathrm{~nm}$ thick $\mathrm{Si}$ continuous layer situated at $3 \mathrm{~nm}$ from the sample surface. In all cases $\mathrm{Ag}^{+}$ions are implanted at $E=$ $10 \mathrm{keV}$ with a fluence of $7.15 \times 10^{15} \mathrm{~cm}^{-2}$, corresponding to 15 at. \% in excess in the $\mathrm{SiO}_{2}$ matrix. The $\mathrm{Si}$ layer used for the simulation in Fig. 2(c) represents a limited case in which a high density of Si-NCs forms a continuous layer. For each condition we have plotted the Si profile before (in blue, up triangles) and after (in black, down triangles) $\mathrm{Ag}^{+}$implantation, and $\mathrm{Ag}$ distribution obtained in the presence of $\mathrm{Si}$ in the layer (in red, full squares). The Ag profile when implanted in pure silica is also plotted (in green, empty squares) for comparison. As it can be noticed, the Si profile is strongly modified by the $\mathrm{Ag}$ implantation; the $\mathrm{Si}$ atoms concentration decreases monotonically for both the Si implanted profile and $\mathrm{Si}$ thin layer due to collisions and mixing phenomena. On the contrary, $\mathrm{Ag}$ atoms distribution in the matrix is the same if the ions are implanted in pure silica, in the presence of either implanted $\mathrm{Si}$ ions or a $\mathrm{Si}$ thin layer, as predicted by TRIDYN the implanted Ag profile does not appear to depend on the matrix history. However, we expect that Ag-NCs distribution will be strongly dependent on the presence of Si-NCs in the matrix and on their density. The experimental results could be different as TRIDYN simulations do not take into account neither the heterogeneous nucleation nor diffusion phenomena that take place during the $\mathrm{Ag}^{+}$implantation.

Figure 3 shows the EFTEM images (filtered at $17 \mathrm{eV}$ ) of Ag-NCs obtained by implanting $\mathrm{Ag}$ at $10 \mathrm{keV}$ with a fluence of $7.125 \times 10^{15} \mathrm{at} \cdot \mathrm{cm}^{-2}$, either in (a) a pure $\mathrm{SiO}_{2}$ matrix (reference sample) or in silica containing Si-NCs with low (b) and high (c) density obtained by ULE-IBS. Low density Si-NCs in (b) have been obtained with $E=1 \mathrm{keV}$ and a
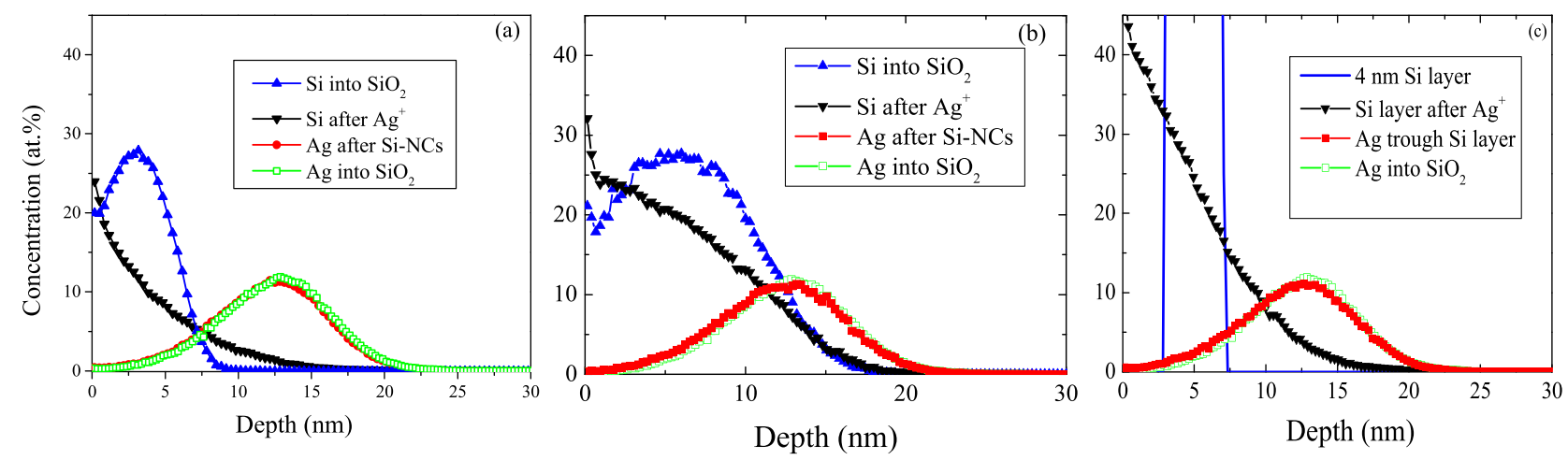

FIG. 2. TRIDYN simulations for $\mathrm{Si}$ and $\mathrm{Ag}$ distribution for (a) $\mathrm{SiO}_{2}$ implanted with $\mathrm{Si}$ at $1 \mathrm{keV}$ and $1 \times 10^{16} \mathrm{~cm}^{-2}$, (b) $\mathrm{SiO} 2$ implanted with $\mathrm{Si}$ at $3 \mathrm{keV}$ and $2 \times 10^{16} \mathrm{~cm}^{-2}$ and (c) $\mathrm{SiO}_{2}$ containing a $4 \mathrm{~nm}$ thick Si continuous (100\% Si) layer situated at 3 nm from the sample surface. In all the three cases Ag ${ }^{+}$ions are implanted at $E=10 \mathrm{keV}$ and with a fluence of $7.15 \times 10^{15} \mathrm{~cm}^{-2}$. Si distribution in pure silica is indicated in blue (up triangles), Si distribution after Ag implantation in black (down triangles), $\mathrm{Ag}$ into $\mathrm{SiO}_{2}$ is green (empty squares) and $\mathrm{Ag}$ after implantation through $\mathrm{Si}-\mathrm{NCs}$ or silicon layer is plotted in red (full squares). 


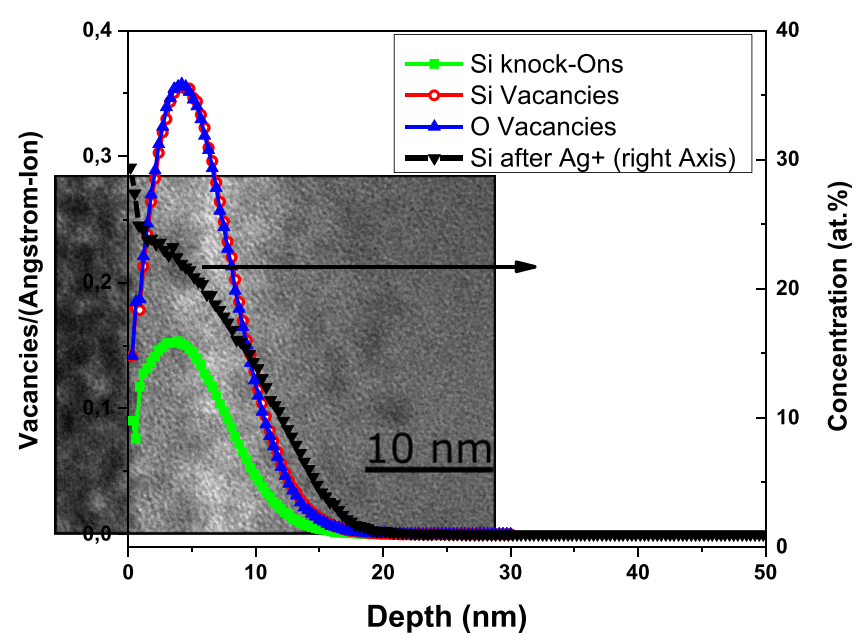

FIG. 3. EFTEM images showing the Ag-NCs distribution obtained by implanting $\mathrm{Ag}$ at $10 \mathrm{keV}$ with a fluence of $7.125 \times 10^{15} \mathrm{at} \cdot \mathrm{cm}^{-2}$, either in (a) a pure $\mathrm{SiO}_{2}$ matrix (reference sample) or in silica containing Si-NCs synthetised at $\mathrm{E}=1 \mathrm{keV}$ and a fluence of $5 \times 10^{15}$ at $\cdot \mathrm{cm}^{-2}$ (b) and with $\mathrm{E}=3 \mathrm{keV}$ and a fluence of $2 \times 10^{16}$ at. $\mathrm{cm}^{-2}(\mathrm{c})$, respectively. The (a) image is a ZeroLoss image, while (b) and (c) are filtered at the Si plasmon (17 eV).

fluence of $5 \times 10^{15}$ at $\cdot \mathrm{cm}^{-2}$ (sample called Ag10Si1), while high density $\mathrm{Si}-\mathrm{NCs}$ in (c) have been synthesized with $E=3 \mathrm{keV}$ and a fluence of $2 \times 10^{16} \mathrm{at} \cdot \mathrm{cm}^{-2}$ (sample called Ag10Si3).

As it can be observed on these image series, our experimental results show that even if TRIDYN predict the same distribution for $\mathrm{Ag}$ ions after the implantation, the resulting Ag-NCs population is strongly dependent on the presence of $\mathrm{Si}-\mathrm{NCs}$ and on their characteristics. Indeed, as expected from previous results, Ag-NCs synthesized in pure silica [Fig. 3(a)] form a 2D array parallel to the sample surface. The distance from the sample surface to the centre of the NCs distribution, as measured on cross sectional TEM (11.6 nm), corresponds to the projected range (Rp) predicted by TRIDYN $(11.3 \mathrm{~nm})$ within the error bar $( \pm 0.5 \mathrm{~nm}) .^{20}$ The $\mathrm{Ag}$-NCs mean size is $5.1 \mathrm{~nm}$. When Ag-NCs are synthesized in presence of a low Si-NCs density [Fig. 3(b)], we observe that a $2 \mathrm{D}$ plane of $\mathrm{Ag}-\mathrm{NCs}$ is still present, and the distance from the sample surface to the centre of the NCs distribution is very close to the previous one $(11.7 \mathrm{~nm})$, even if the nanocrystals mean diameter is bigger $(8.8 \mathrm{~nm})$. In addition, small Ag-NCs (2.6 nm of mean diameter) located deeper in the silica matrix and widely distributed can be observed. We do not observe any $\mathrm{Si}-\mathrm{NC}$ after Ag implantation in EFTEM images. This is probably due to the ion mixing during Ag implantation. When $\mathrm{Si}$ atoms have been displaced then mixed $\mathrm{Si}-\mathrm{NCs}$ and $\mathrm{Si}$ atoms or $\mathrm{Si}$ small clusters cannot be imaged in TEM.

For a high density of Si-NCs sample [Fig. 3(c)], the 2D array of Ag-NCs is replaced by a broad band of small AgNCs. The Si rich zone is visible at the same location as the previous $\mathrm{Si}-\mathrm{NCs}$.

The understanding of the differences found in the AgNCs distribution for these 3 cases is not trivial. As already noticed, several phenomena can take place during Ag-NCs synthesis. The two main routes we explored are (1) the modified diffusion of $\mathrm{Ag}$ into the matrix containing $\mathrm{Si}-\mathrm{NCs}$ (or $\mathrm{Si}$ excess) with respect to a pure silica matrix, and (2) the heterogeneous nucleation on defects such as oxygen and silicon vacancies due to the previous $\mathrm{Si}$ ion implantation or in $\mathrm{Si}$ excess itself.

\section{Modified Ag diffusion with Si content in Si-rich silica matrix}

In order to elucidate the observed behaviour, let us first discuss the impact of the presence of Si-NCs (or of a $\mathrm{Si}$ excess) in the matrix on Ag diffusion and Ag-NCs synthesis.

Following Strobel, ${ }^{21}$ the nucleation process during ion beam synthesis depends on the value of the diffusion length $L_{\text {Diff }}=\sqrt{2 D t}$ of the impurity ions during the implantation (where $t$ is the implantation time and $D$ is the diffusion coefficient) compared to the width of the ion distribution profile due to straggling $\Delta \mathrm{Rp}$, and to the critical radius $\widehat{R}$ for a given implanted fluence.

- When $\mathrm{L}_{\text {Diff }}<\widehat{R}$ thermal fluctuations cannot initiate the nucleation and thermal annealing is needed to obtain NCs. This is the case, for example, for Si-NCs IBS in silica.

- When $\mathrm{L}_{\text {Diff }}>\widehat{R}$, nucleation starts during implantation when the atom concentrations exceeds the nucleation threshold. This is usually met for metal implantation into the insulators. In addition, if $\mathrm{L}_{\text {Diff }} \approx \Delta \mathrm{Rp}$, the NCs are formed across the whole deposition range with a density proportional to the impurity profile, and if $\mathrm{L}_{\text {Diff }} \gg \Delta \mathrm{Rp}$, the NCs are formed close to the Rp. Indeed, in this last case, the NCs formation starts close to Rp, where the highest concentration of impurities is present. Due to the high mobility of the impurities, during the implantation, the atoms diffuse from the tail of the distribution toward the centre, where the already formed NCs act as sinks.

In our study, the ULE implantation in a silica matrix of $\mathrm{Ag}$ ions and with the corresponding ions fluencies generate systems which behaviour is well described by the case in which $\mathrm{L}_{\text {Diff }} \gg \Delta \mathrm{Rp}>\widehat{R}$. Indeed, $\mathrm{Ag}$-NCs are formed during the implantation and are distributed in a $2 \mathrm{D}$ array in the zone close to the Rp.

On the other side, when Si-NCs where previously synthesised in the same matrix, the system behaviour for the same implantation conditions changes and the 2D array of $\mathrm{Ag}-\mathrm{NCs}$ close to $\mathrm{Rp}$ is no more formed. The formation of $\mathrm{Ag}-\mathrm{NCs}$ in the whole deposition range seems to suggest a shorter diffusion length for $\mathrm{Ag}$ ions in the Si-rich matrix. We have calculated $\mathrm{L}_{\text {diff }}$ by using the values of $\mathrm{D}$ at $300^{\circ} \mathrm{C}$ from McBrayer et al. ${ }^{22}$ for the diffusion of $\mathrm{Ag}$ in silica $\left(\mathrm{D}_{\mathrm{Ag} / \mathrm{SiO} 2}\right.$ $=10^{-13} \mathrm{~cm}^{2} / \mathrm{s}$ ) and from Coffa et al. ${ }^{23}$ for Ag in amorphous silicon $\left(\mathrm{D}_{\mathrm{Ag} / \mathrm{Si}}=4.5 \times 10^{-16} \mathrm{~cm}^{2} / \mathrm{s}\right)$ respectively. As the time $\mathrm{t}$ for implantation in this case is around $3 \mathrm{~h}$, we have obtained for $\mathrm{L}_{\text {Diff }}=\sqrt{2 D t}$ in silica and in amorphous silicon, $500 \mathrm{~nm}$ and $33 \mathrm{~nm}$, respectively. This rough calculation shows that the diffusion length in silica is 15 times higher than in amorphous silicon. Of course, the D value can in principle be lower if the real temperature during ion implantation is less than $300{ }^{\circ} \mathrm{C}$, and in this case, $\mathrm{L}_{\text {Diff }}$ would be shorter too. The value of $\Delta R_{p}$ calculated by TRIM in our implantation conditions is about $3 \mathrm{~nm}$. 
These values are in agreement with the observed behaviour in our system, in fact, Ag-NCs nucleation in silica $\left(\mathrm{L}_{\text {Diff }}=500 \mathrm{~nm} \gg \Delta \mathrm{Rp}=3 \mathrm{~nm}\right)$ takes place in a region restricted close to the $\mathrm{Rp}$, in agreement with the observed distribution of nanocrystals [Fig. 3(a)].

On the other hand, when a high Si excess is present in silica, the diffusion length of the host material changes and decreases towards the one found in amorphous silicon $(33 \mathrm{~nm})$. This decrease of the diffusion length with respect to pure silica, when a high density of Si-NCs is present in the matrix could in principle be responsible for a change in the nucleation regime. In fact, $\mathrm{Ag}-\mathrm{NCs}$ distribution is larger [Fig. 3(c)] and the nucleation takes place in the entire implantation region. An intermediate situation is found for the sample with low Si excess [Fig 3(b)].

However, this modified diffusion cannot be responsible only for differences observed in the synthesis of Ag-NCs following the Si content in the matrix. In fact, when the Si content in the matrix is high (sample $\mathrm{Ag} 10 \mathrm{Si} 3$ ) the nanocrystals are not formed with a density roughly proportional to the impurity profile, as it is expected in case of $\mathrm{L}_{\text {Diff }} \approx \Delta \mathrm{Rp}$, but Ag-NCs [Fig. 3(c)] are uniformly distributed in a broad band.

Consequently, other phenomena should be coresponsible for the final distribution of $\mathrm{Ag}-\mathrm{NCs}$ in double layers synthesis by ULE-IBS.

\section{Heterogeneous nucleation}

In order to better understand the evolution of our system when increasing the implanted Si content, the heterogeneous nucleation has to be taken into account. Indeed, it is well known that the heterogeneities present in the matrix, such as grain boundaries, impurities or defects can also catalyse the phase transition and that the effective nucleation rate can differ from the homogeneous nucleation.

Let us now discuss the possible origin of these nucleation centres.

First of all, the defects induced by $\mathrm{Si}$ ion implantation can still be present in the matrix even after a $1050{ }^{\circ} \mathrm{C}$ RTA annealing and induce Ag clustering. However, if heterogeneous nucleation due to implantation defects has a major influence, the final Ag-NCs distribution should be correlated to the damage profile, which is not found in our case. Indeed, if we superimpose TEM images of our samples and the defects profile calculated by SRIM, we can notice that silicon and oxygen vacancies due to $\mathrm{Si}$ ion implantation are located in the zone between the surface and the Rp of the implanted Si (corresponding to Si-NCs position after annealing). An example is shown in Fig. 4 for the sample Ag10Si3. Consequently, we can conclude that this kind of defect plays no role on the Ag-NCs nucleation.

Other defects such as the matrix porosity could be responsible for the loss of the 2D distribution in ULE-IBS. Indeed, previous studies have shown that humidity from the ambient air can penetrate the damaged or porous layers after implantation. ${ }^{24}$ We have also observed (not shown here) that the $\mathrm{Ag}$ ions distribution is wider in a porous layer (porosity due to ion implantation or to the matrix deposition method) than in a pure thermal silica and results in a broader distribution of nanocrystals. In our case, we can neglect the effect of the matrix porosity due to the damage of $\mathrm{Si}^{+}$implantation as a high temperature annealing has been performed before $\mathrm{Ag}$ NCs synthesis, which induces a good recovering of the matrix integrity.

The most probable heterogeneous nucleation centres are $\mathrm{Si}$ atoms in excess in the matrix that can play the role of catalyser for Ag-NCs nucleation. Indeed, small $\mathrm{Ag}-\mathrm{NCs}$ observed in the Ag10Si3 and Ag10Si1 samples can be formed by the heterogeneous nucleation in $\mathrm{Si}$ excess or in small $\mathrm{Si}$ clusters that are present in the matrix after the SiNCs mixing due to the Ag implantation. Fig. 4 also shows the $\mathrm{Si}$ distribution after $\mathrm{Ag}$ implantation according to TRIDYN simulations in the case of the sample Ag10Si3, superposed to the EFTEM image of this sample.

As a summary following these considerations, we can explain the Ag-NCs distribution in the samples implanted with $\mathrm{Ag}^{+}$at $10 \mathrm{keV}$ in samples containing small (Ag10Si1) or high (Ag10Si3) amount of Si-NCs, respectively, with respect to the reference sample containing Ag-NCs in silica in the following way: in the first case $\mathrm{Si}$ is implanted at $1 \mathrm{keV}$, very close to the surface sample (distance of Si-NCs to surface $=2.8 \mathrm{~nm}$ ) and a low density of Si-NCs is formed after annealing. Subsequent Ag implantation mixes the SiNCs layer and Si atoms are redistributed. As Si concentration is low, the Ag-NCs nucleation regime remains very similar to the case of $\mathrm{Ag}-\mathrm{NCs}$ in pure silica, but $\mathrm{Si}$ atoms distributed in the matrix can play the role of sparse nucleation sites for heterogeneous nucleation in the region where the $\mathrm{Si}$ excess is distributed after the Ag-NCs principle plane, producing the small Ag-NCs population. In the second case (Ag10Si3), $\mathrm{Si}$ is implanted with a higher fluence and $\mathrm{Si}-\mathrm{NCs}$ form a dense array of percolated Si-NCs and after ion mixing due to $\mathrm{Ag}$ implantation the matrix is rich in Silicon, which modify the diffusion regime on the one side, and on the other side
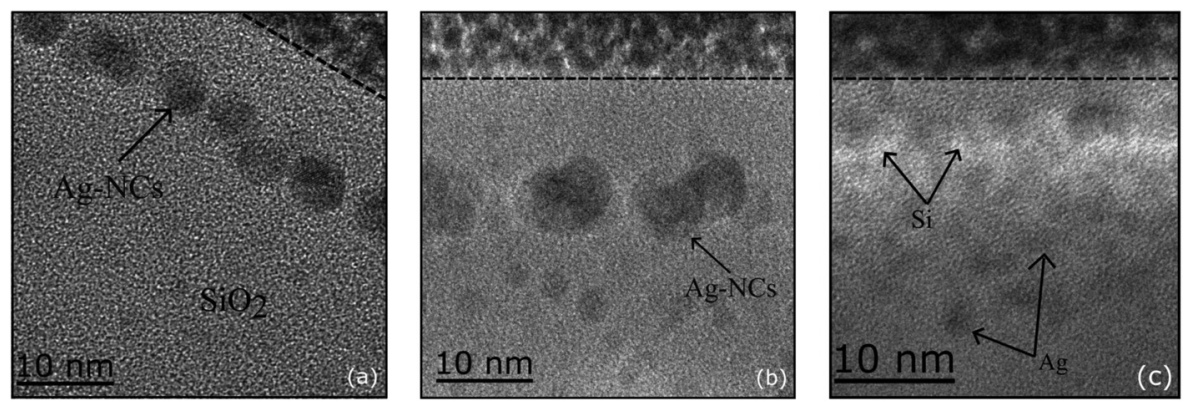

FIG. 4. Cross sectional EFTEM image of the sample $\mathrm{Ag} 10 \mathrm{Si} 3$, superposed to the distribution of Si knock-ons, $\mathrm{Si}$ and $\mathrm{O}$ vacancies (left scale) as calculated by TRIM, as well as to the $\mathrm{Si}$ ions distributions after $\mathrm{Ag}+$ implantation calculated by TRIDYN (right scale). 
allows heterogeneous nucleation of $\mathrm{Ag}-\mathrm{NCs}$ in the whole region of Si excess distribution.

Another parameter, which has to be taken into account, is the nitrogen diffusion in the matrix during the RTA annealing at $1050^{\circ} \mathrm{C}$ under $\mathrm{N}_{2}$ atmosphere for the Si-NCs nucleation. Indeed, the presence of $\mathrm{N}$ atoms in the matrix can either modify the Ag diffusion in the matrix ( $\mathrm{SiNx}$ matrix is known to be a barrier for $\mathrm{Ag}$ diffusion ${ }^{25,26}$ ) or act as heterogeneous nucleation centres. At the moment we do not have any evidence of the presence of nitrogen in our matrix, as the Fourier Transform Infra Red spectroscopy (FTIR) analysis of the layers did not show any nitrogen presence. However, very small amounts of nitrogen atoms, undetectable with this method could be present in the layers. For this reason, further experiments with thermal annealing under $\mathrm{Ar}$ instead of $\mathrm{N}_{2}$ have been planned, in order to elucidate the role of nitrogen in the Ag-NCs nucleation in Si rich silica.

Unhappily, these results shows that due to the necessity to synthesize Si-NCs first, it is impossible to obtain two separated layers of nanocrystals by using 1 to $3 \mathrm{keV} \mathrm{Si}$ ions and $10 \mathrm{keV} \mathrm{Ag}$ ions.

Our strategy to obtain double 2D-layers is to reduce the $\mathrm{Si}$-NCs ion mixing, which seems to be mainly responsible for the lack of the planar distribution of Ag-NCs. For this, the the method of exploration in this work has been to reduce Ag implantation energy to $3 \mathrm{keV}$.

\section{B. The case of Ag-NCs synthesized at $E=3 \mathrm{keV}$}

In this case, TRIDYN simulations (Fig. 5) give a Si profile in the region of Si-NCs, which is nearly Gaussian after a $3 \mathrm{keV} \mathrm{Ag}$ implantation, indicating that the ion mixing phenomenon is reduced.

As the silver implantation energy is decreased, the implanted fluence is also reduced in order to maintain the

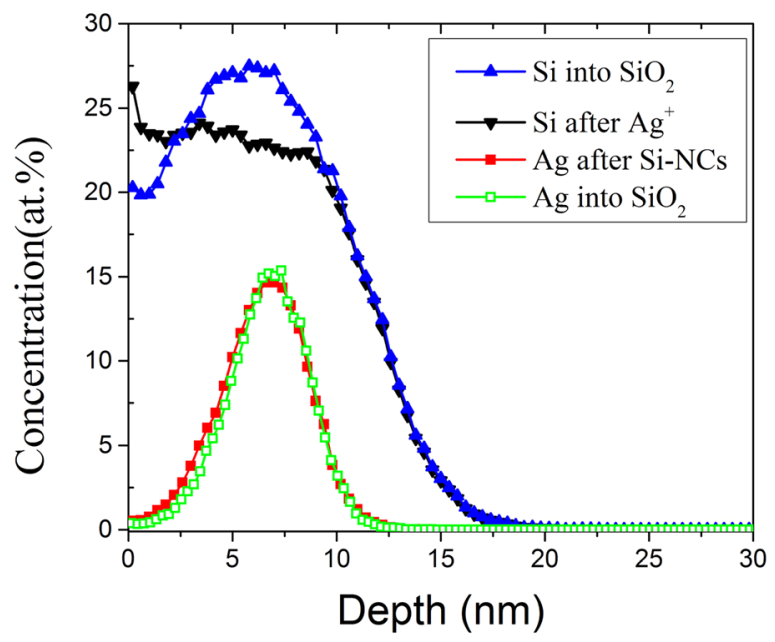

FIG. 5. TRIDYN simulations for $\mathrm{Si}$ and $\mathrm{Ag}$ distribution in the conditions used for the sample $\mathrm{Ag} 3 \mathrm{Si} 3: \mathrm{SiO}_{2}$ is implanted with $\mathrm{Si}$ at $3 \mathrm{keV}$ and $2 \times 10^{16} \mathrm{~cm}^{-2}$, then $\mathrm{Ag}^{+}$ions are implanted at $\mathrm{E}=3 \mathrm{keV}$ and with a fluence of $4.7 \times 10^{15} \mathrm{~cm}^{-2}$. Si distribution in pure silica is indicated in blue (up triangles), Si distribution after $\mathrm{Ag}$ implantation in black (down triangles), $\mathrm{Ag}$ into $\mathrm{SiO}_{2}$ is green (empty circles), and $\mathrm{Ag}$ after implantation through $\mathrm{Si}-\mathrm{NCs}$ or silicon layer is plotted in red (full squares).
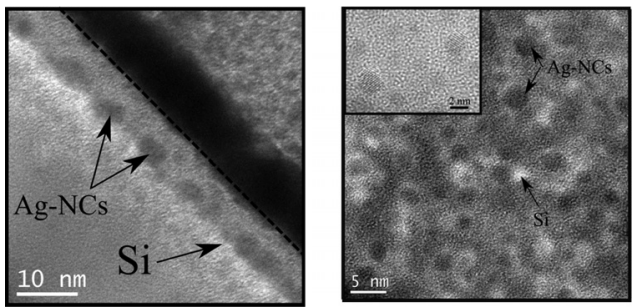

FIG. 6. EFTEM images of the sample $\mathrm{Ag} 3 \mathrm{Si} 3$ obtained by filtering around the Si plasmon, in cross section (a) and in plain view (b), respectively. The inset corresponds to an HREM image in plain view. The synthesis conditions are indicated in the caption of the Fig. 5.

same Ag concentration at Rp. Having a lower fluence leads to less damage and ion mixing. In this case, the implanted Ag fluence is reduced to $4.7 \times 10^{16} \mathrm{~cm}^{-2}$, corresponding to 20 at. \% at Rp. Concerning Si-NCs synthesis, we have used the same conditions as for previous sample with a high density Si-NCs, i.e., $3 \mathrm{keV}$ energy and $2 \times 10^{16} \mathrm{~cm}^{-2}$, with a $1050^{\circ} \mathrm{C}$ RTA annealing during $60 \mathrm{~s}$ under $\mathrm{N}_{2}$ ambience. Figure 6(a) shows a cross section EFTEM image of this sample, called Ag3Si3. Two distinct thin layers can be observed in the image: Ag-NCs, with a dark contrast, are easily distinguishable. Another zone with white contrast indicates that pure silicon is present and forms a $2.3 \mathrm{~nm}$ thick layer in the $\mathrm{SiO}_{2}$ very close to Ag-NCs. Due to the projection of several particles in the observed zone, this kind of image does not allow saying if this layer is composed of Si nanoparticles or if the white zone corresponds to an amorphous silicon layer. For this, we have performed plain view EFTEM experiments, which are shown in Fig. 6(b). As we can notice, AgNCs are well separated, crystallized (see inset) and their mean size is $2.6 \mathrm{~nm}$. On the other hand, after Ag implantation, silicon does not form a continuous layer but forms separated $\mathrm{Si}$ clusters with a mean size of $2.3 \mathrm{~nm}$. These clusters are amorphous; no Si crystalline plane has been observed in HREM.

In order to compare the Si nanoclusters evolution due to $\mathrm{Ag}^{+}$ion implantation, the Fig. 7 shows EFTEM images in cross section (a) and in plain view (b) of the sample before Ag-NCs synthesis. It can be noticed that $\mathrm{Si}-\mathrm{NCs}$ synthesised in the first step of the double layers fabrication $\left(\mathrm{Si}^{+} 3 \mathrm{keV}, 2\right.$ $\times 10^{16} \mathrm{ions} / \mathrm{cm}^{2}$ ) form a $2 \mathrm{D}$ array of crystallized and coalesced nanocrystals, with a surface coverage of $83 \%$. This means that Ag-NCs synthesis modifies the Si-NCs population even if we have limited the amount of ion mixing.
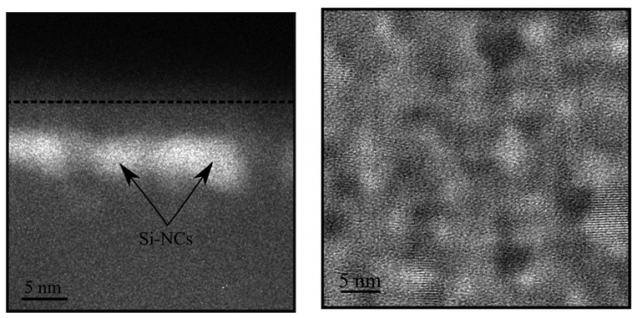

FIG. 7. EFTEM images of the Si-NCs obtaining by implanting $\mathrm{Si}^{+}$at $3 \mathrm{keV}$ and with a dose of $2 \times 10^{16}$ ions $/ \mathrm{cm}^{2}$, in cross section (a) and in plain view (b), respectively. In the plain view image, we can notice that Si nanostructures are crystallized. 
Indeed, the high-density array of coalesced and crystalline Si-NCs is changed in a 2D array of separated and amorphous nanoparticles due to $\mathrm{Ag}^{+}$ion implantation and subsequent ion mixing.

The results shown in the Fig. 6 demonstrate that using lower energy for Ag implantation, it is possible to synthesize two arrays of Si nanoparticles and Ag-NCs. This is a major result because it shows for the first time that the elaboration of 2D arrays of different nanoparticles species only by ULEIBS is possible by minimizing the ion-mixing phenomena and by taking into account the differences on thermal budgets for nanocrystals synthesis. At the moment, both obtained nanoparticles layers are very close, as it was expected following the TRIDYN simulation, which is not an ideal configuration for Si-NCs PL enhancement. Indeed, Anger et al. ${ }^{27}$ have shown that PL enhancement due to plasmonic nanoparticles has a maximum for an optimum distance to the emitter, but fluorescence is quenched for shorter distances. Several parameters might be explored to control the bi-layers synthesis and to find optimum configurations. Indeed, work is in progress in order to control the distance of both layers through the study of annealing treatments before and after the synthesis of Ag-NCs. Concerning the amorphous state of the Si nanoclusters after the synthesis of Ag-NCs, some preliminary experience have shown that in such hors equilibrium systems, it is possible to obtain the recrystallization of Si nanoparticles by annealing the whole system at $600{ }^{\circ} \mathrm{C}$ for a short time $(60 \mathrm{~s})$ and this, without reducing the $\mathrm{Ag}$ content in the Ag-NCs. However, even if the obtained bi-layers are not yet optimised, the first results obtained for PL emission are encouraging.

\section{PL emission properties of the sample Ag3Si3}

Figure 8 shows the PL properties of Si nanocrystals with or without the presence of Ag-NCs. In order to distinguish the two cases and directly compare the effect of Ag-NCs on PL efficiency, a zone of the sample Ag3Si3 has been covered with a stencil mask before Ag ion implantation. The zone B in Fig. 8 is located in a part of the sample masked after Si-NCs synthesis, while the zone A has been implanted with $\mathrm{Ag}^{+}$ions in order to obtain Ag-NCs. The image in Fig. 8(a) has been obtained by integrating the light emission between 600 and $750 \mathrm{~nm}$ : one clearly observes a strong exaltation of the Si-NCs PL induced by the presence of silver. Figure 8(b) shows the spectra obtained by averaging the signal from zones A and B. As we can notice, a typical PL emission from Si nanoparticles, centred at $670 \mathrm{~nm}$, is only observed in the A zone, containing both $\mathrm{Si}$ nanostructures and $\mathrm{Ag}$ nanoparticles. This behaviour can be explained if we consider that in the zone B the Si-NCs are present with a high density (83\% surface covering) and form a percolated structure (see Fig. 7) resulting in the loss of quantum confinement and thus of PL emission. On the contrary, in the A zone, Si-NCs have evolved in separated $\mathrm{Si}$ nanoparticles with small size $(2.3 \mathrm{~nm})$ due to partial mixing after Ag ion implantation, which could favour the quantum confinement and then, the PL emission. However, as already mentioned, we could not observe any crystalline silicon nanoparticle by HREM after Ag-NCs formation, meaning that $\mathrm{Si}$ nanoparticles in the obtained bi-layers are mainly amorphous. It is well known that PL emission from amorphous Si nanoparticles with respect to the crystalline ones is very weak. Indeed, as an example, Anthony and Kortshagen ${ }^{28}$ have shown that the quantum yield of amorphous nanoparticles is $2 \%$, while the quantum yield of silicon nanocrystals exceed $40 \%$. From the value of the maximum of the PL $(670 \mathrm{~nm}$, i.e., $1.85 \mathrm{eV})$, one can deduce a corresponding average size of amorphous $\mathrm{Si}$ quantum dots around $2.5 \mathrm{~nm},{ }^{29}$ in rather good agreement with our plain view TEM observations. Consequently, we suggest that the Si nanoparticles photoluminescence observed in our sample comes from small amorphous $\mathrm{Si}$ dots. We can guess that the relatively high intensity of this PL emission could be due to plasmonic effect of Ag-NCs. Indeed, even if the two particles layers are very close, the distances between Si nanoclusters and Ag-NCs on the plane could allow their coupling. To conclude, the exaltation of the PL emission from zone A in Fig. 8 is induced by the Ag-NCs synthesis process. Two reasons can be put forward: the structural modification of the Si$\mathrm{NCs}$ induced by $\mathrm{Ag}^{+}$implantation and/or the plasmonic
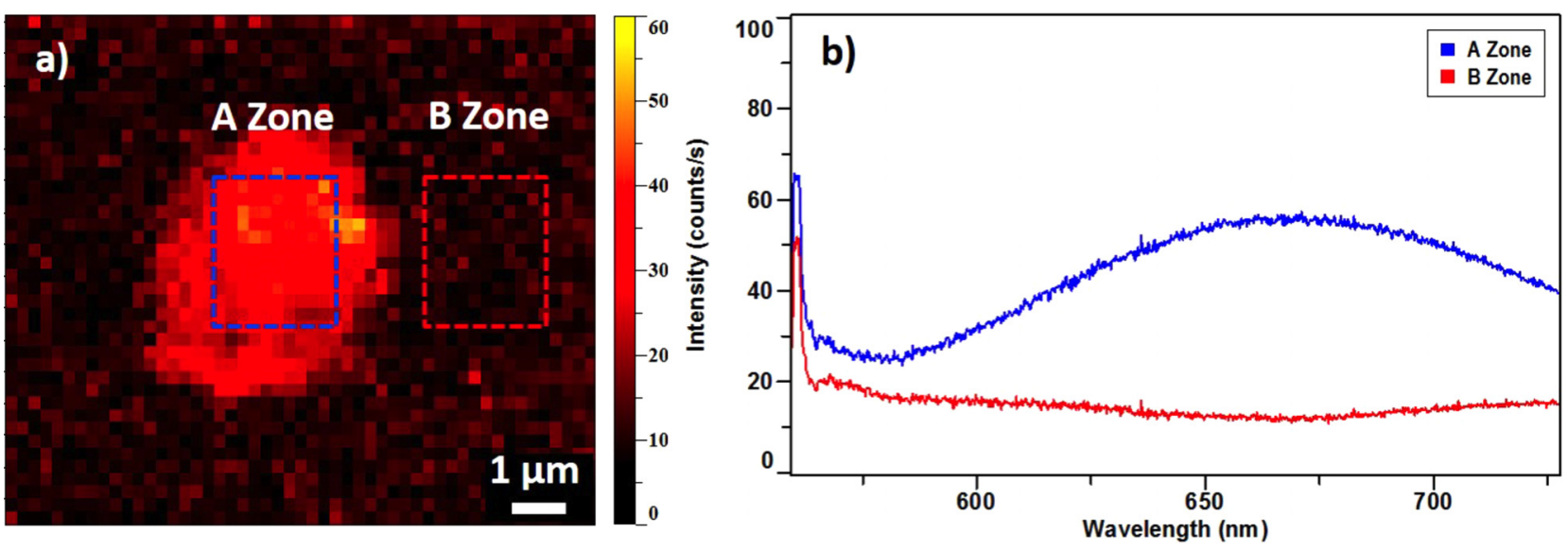

FIG. 8. Photoluminescence of the sample Ag3Si3 obtained at room temperature using a 532 nm laser line as excitation: (a) X-Y map of the emission intensity obtained by integrating the signal between 600 and $750 \mathrm{~nm}$ and using a step scan of $0.2 \mu \mathrm{m}$; (b) photoluminescence spectra obtained by averaging the signal coming from Si-NCs in the presence (A Zone) or the absence (B Zone) of Ag-NCs. The peak located near $560 \mathrm{~nm}$ corresponds to the second order Raman signal originating from the Si substrate. 
coupling due to the presence of Ag-NCs. However, further investigations are necessary in order to clarify this point.

The aim of this work was to investigate the ability to fabricate double 2D layers of Si- and Ag-NCs by ULE-IBS and to finely control their characteristics. These results show that 2D arrays of amorphous Si nanoparticles and $\mathrm{Ag}-\mathrm{NCs}$ can be synthesised by ULE-IBS and that controlling their size and mutual distance at the nanoscale is very promising for Si-NCs PL enhancement. These structures are suitable for light conversion layers application in third generation photovoltaics. Indeed, strong Si-NCs PL enhancement should, in principle, be achieved after the optimisation of the design of the composite system.

For this, several routes are explored in order to obtain two well-separated 2D layers of Si and Ag-NCs, including the implantation through aligned stencil mask of nanometric size, the use of silicon nitride intermediate layers, and the optimisation of the intermediate annealing (ambient and thermal budget) before $\mathrm{Ag}^{+}$implantation. Some preliminary results show that the controlled separation of the layers can be obtained through an appropriate annealing process before Ag-NCs synthesis, which allows obtaining $\mathrm{Si}-\mathrm{NCs}$ in a deeper position in the matrix.

\section{CONCLUSIONS}

In this paper, we have explored the feasibility to obtain 2D networks of Si-NCs and $\mathrm{Ag}-\mathrm{NCs}$ in a dielectric matrix by Ultra-Low-Energy Ion-Beam-Synthesis. For this, we have first synthesised 2D layers of Si-NCs by $\mathrm{Si}^{+}$ion implantation at ultra low energy followed by a high temperature thermal annealing. Then, $\mathrm{Ag}^{+}$ions have been implanted at energies between 3 and $10 \mathrm{keV}$ leading to the synthesis of an array of Ag-NCs. We have shown that several physical phenomena are simultaneously involved in the synthesis of the double layer hybrid system and include ion mixing, implantation damage, point defect, and thermal diffusion.

The Ag-NCs synthesis in matrixes containing Si-NCs has been discussed in terms of nucleation regime and thermal diffusion, as well as heterogeneous nucleation. Our results show that it is possible to obtain 2D arrays of Si nanoparticles and Ag nanocrystals if the amount of ion mixing is low, which is the case when implanting ions $\mathrm{Ag}^{+}$at very low energy (around $3 \mathrm{keV}$ ). In these conditions, a PL enhancement has been observed when Si nanoclusters are located at the vicinity of the Ag-NCs. A stronger PL enhancement is expected by optimizing the size, crystallinity, and mutual distance of the embedded arrays of NCs.

\section{ACKNOWLEDGMENTS}

The authors are grateful to the French National Research Agency that provided support for this work through the GENESE Project (No. ANR-13-BSS09-002001).
${ }^{1}$ T. Trupke, M. A. Green, and P. Würfel, J. Appl. Phys. 92, 1668 (2002).

${ }^{2}$ M. A. Green, Third Generation Photovoltaics: Advanced Solar Energy Conversion (Springer, Berlin, Heidelberg, Germany, 2003).

${ }^{3}$ J. S. Biteen, L. A. Sweatlock, H. Mertens, N. S. Lewis, A. Polman, and H. A. Atwater, J. Phys. Chem. C 111, 13372 (2007).

${ }^{4}$ K. R. Catchpole and A. Polman, Opt. Express 16, 21793 (2008).

${ }^{5}$ H. A. Atwater and A. Polman, "Plasmonics for improved photovoltaics devices,” Nat. Mater. 9, 205 (2010).

${ }^{6}$ T. Nychyporuk, Y. Zakharko, T. Serdiuk, O. Marty, M. Lemiti, and V. Lysenko, Nanoscale 3, 2472 (2011).

${ }^{7}$ S.-K. Kim, C.-H. Cho, B.-H. Kim, Y.-S. Choi, S.-J. Park, K. Lee, and S. Im, Appl. Phys. Lett. 94, 183108 (2009).

${ }^{8}$ H. Mertens, J. S. Biteen, N. S. Lewis, H. A. Atwater, and A. Polman, "Polarization-selective plasmon-enhanced silicon quantum-dot luminescence," Nano Lett. 6, 2622 (2006).

${ }^{9}$ J. S. Biteen, N. S. Lewis, H. A. Atwater, H. Mertens, and A. Polman, Appl. Phys. Lett. 88, 131109 (2006).

${ }^{10}$ J. S. Biteen, D. Pacifici, N. S. Lewis, and H. A. Atwater, Nano Lett. 5, 1768 (2005).

${ }^{11}$ J. Kalkman, H. Gersen, L. Kuipers, and A. Polman, Phys. Rev. B 73, 075317 (2006).

${ }^{12}$ A. Benami, A. Lopez-Suarez, L. Rodriguez-Fernandez, A. Crespo-Sosa, J. C. Cheang-Wong, J. A. Reyes-Esqueda, and A. Oliver, AIP Adv. 2, 012193 (2012).

${ }^{13}$ J. Bornacelli, J. A. Reyes Esqueda, L. Rodríguez Fernández, and A. Oliver, J. Nanotechnol. 2013, 736478.

${ }^{14}$ C. Bonafos, M. Carrada, N. Cherkashin, H. Coffin et al., J. Appl. Phys. 95, 5696 (2004)

${ }^{15}$ C. Bonafos, H. Coffin, S. Schamm, N. Cherkashin, G. Ben Assayag, P. Dimitrakis, P. Normand, M. Carrada, V. Paillard, and A. Claverie, SolidState Electron. 49, 1734 (2005).

${ }^{16}$ G. Ben Assayag, C. Bonafos, M. Carrada, A. Claverie, P. Normand, and D. Tsoukalas, Appl. Phys. Lett. 82, 200 (2003).

${ }^{17}$ M. Carrada, N. Cherkashin, C. Bonafos, G. Benassayag, D. Chassaing, P. Normand, D. Tsoukalas, V. Soncini, and A. Claverie, Mater. Sci. Eng., B 101, 204 (2003).

${ }^{18}$ R. Carles, C. Farcau, C. Bonafos, G. Benassayag, B. Pécassou, and A. Zwick, Nanotechnology 20, 355305 (2009).

${ }^{19}$ P. Benzo, C. Bonafos, M. Bayle, R. Carles, L. Cattaneo, C. Farcau, G. Benassayag, B. Pécassou, and D. Müller, J. Appl. Phys. 113, 193505 (2013).

${ }^{20}$ S. Schamm, C. Bonafos, H. Coffin, N. Cherkashin, M. Carrada, G. Ben Assayag, A. Claverie, M. Tencé, and C. Colliex, Ultramicroscopy 108, 346 (2008).

${ }^{21}$ M. Strobel, Ph.D. thesis, Technische Universitat Dresden, Forschungszentrum Rossendorf, Wissenschaftlich-Technische Berichte FZR-277, 1999.

${ }^{22}$ J. D. McBrayer, R. M. Swanson, and T. W. Sigmon, J. Electrochem. Soc. 133, 1242 (1986).

${ }^{23}$ S. Coffa, J. M. Poate, D. C. Jacobson, W. Frank, and W. Gustin, Phys. Rev. B 45, 8355 (1992).

${ }^{24}$ A. Claverie, C. Bonafos, G. Ben Assayag, S. Schamm, N. Cherkashin, V. Paillard, P. Dimitrakis, E. Kapetenakis, D. Tsoukalas, T. Muller, B. Schmidt, K. H. Heinig, M. Perego, M. Fanciulli, D. Mathiot, M. Carrada, and P. Normand, Diffus. Solids Liq. Defects Diffus. Forum 258-260, 531 (2006).

${ }^{25}$ J. Hoornstra, G. Schubert, K. Broek, F. Granek, and C. LePrince, "In lead free metallisation paste for crystalline silicon solar cells: From model to results," in Proceedings of the Photovoltaic Specialists Conference (IEEE, 2005), p. 1293.

${ }^{26}$ Q. A. Acton, Heavy Metals-Advances in Research and Application (Scholarly Editions, Atlanta, GA, 2013).

${ }^{27}$ P. Anger, P. Bharadwaj, and L. Novotny, Phys. Rev. Lett. 96, 113002 (2006).

${ }^{28}$ R. Anthony and U. Kortshagen, Phys. Rev. B 80, 115407 (2009).

${ }^{29}$ N.-M. Park, T.-S. Kim, and S.-J. Park, "Band gap engineering of amorphous silicon quantum dots for light-emitting diodes," Appl. Phys. Lett. 78, 2575 (2001). 